\title{
UNATTAINED BOUNDARY POINTS OF THE NUMERICAL RANGE OF HILBERT SPACE OPERATORS
}

\author{
SRIMATI MAJumdar
}

The numerical range of an operator on a complex Hilbert space is considered and we introduce various known results associated with different points of the numerical range. By the Toeplitz-Hausdorff theorem, the numerical range is a convex set in the complex plane, though not necessarily closed. Thus these results, though interesting, are inapplicable to the unattained boundary points of the numerical range. Therefore, generalizations of these results which may hold for all points of the closure of the numerical range seem to be called for.

We follow the definitions of Embry [6] for certain subsets consisting of vectors from the Hilbert space and then extend these definitions to subsets consisting of bounded sequences of vectors. As observed in this thesis, these two types of subsets are very similar in properties. Embry has characterized the numerical range by means of subsets of vectors and we attempt to characterize the closure of the numerical range by means of subsets consisting of sequences. These characterizations, though similar, are not exactly alike. For example, the linearity of the subset still holds when the point associated with it is an extreme point of the closure of the numerical range, but for nonextreme boundary points, the expression for the linear span of the associated subset is somewhat different from that given by Embry.

Received 14 January 1985. Thesis submitted to University of New England, October, 1983. Degree approved October 1984. Supervisor: Dr Brailey Sims.

Copyright Clearance Centre, Inc. Serial-fee code: 0004-9727/85 $\$ \mathrm{~A} 2.00+0.00$. 
To prove some of these results we use a modification of a technique developed by Berberian [2,3], which involves a change of Hilbert space and operator via a construction based on normalized positive linear functionals. This technique has proved very useful. Throughout our dissertation we will make use of it to obtain generalizations of results by interpreting the results themselves in the new space and operator.

We extend two Cauchy-Schwartz type inequalities of Embry [8] to the unattained boundary points of the numerical range and obtain a result by Das and Craven [5] as a corollary. The orthogonal tendency of sequences from subsets associated with the boundary is also noted.

Embry [7] has shown that the subsets associated with points of the numerical range behave in a particular fashion if the operator has special characteristics and vice versa. We achieve some easy generalizations of these results for subsets consisting of sequences. Several results of Lin [11], Stampfli [12] and de Barra [1] concerning seminormal and convexoid operators are then extended to the unattained boundary points of the numerical range.

Das [4] and Garske [9] gave a theorem concerning weak convergence to zero at the unattained extreme points of the closure of the numerical range. Das and Craven [5] also gave a bound for the norm of the weak limit of sequences corresponding to points on a line segment on the boundary of the numerical range. We achieve all these results as a simple corollary of a generalized Cauchy-Schwartz inequality.

In the concluding part of our thesis we investigate whether convexity holds for other numerical ranges as well. A restricted numerical range is defined and certain conditions are imposed so that this newly defined numerical range is convex. As a corollary to this result, we deduce the convexity of Stampfli's numerical range [13], a result proved differently by Kyle [10].

\section{References}

[1] G. de Barra, "The boundary of the numerical range", Glasgow Math. J. $22(1981), 69-72$. 
Unattained boundary points of the numerical range 469

[2] S.K. Berberian, "Approximate proper vectors", Proc. Amer. Math. Soc. 13 (1962), 111-114.

[3] S.K. Berberian and G.H. Orland, "On the closure of the numerical range of an operator", Proc. Amer. Math. Soc. 18 (1967), 499-503.

[4] K.C. Das, "Boundary of numerical range", J. Math. Anal. Appl. 60 (1977), 779-780.

[5] K.C. Das and B.D. Craven, "Linearity and weak convergence on the boundary of numerical range", J. Austral. Math. Soc. Ser. A 35 (1983), 221-226.

[6] M.R. Embry, "The numerical range of an operator", Pacific J. Math. 32 (1970), 647-650.

[7] M.R. Embry, "Classifying special operators by means of subsets associated with the numerical range", Pacific J. Math. 38 (1971), 61-65.

[8] M.R. Embry, "Orthogonality and the numerical range", J. Math. Soc. Japan 27 (1975), 405-411.

[9] Gerhard Garske, "The boundary of the numerical range of an operator", J. Math. Anal. Appl. 68 (1979), 605-607.

[10] J. Kyle, "W ${ }_{\delta}(T)$ is convex", Pacific J. Math. 72 (1977), 483-485.

[11] C.S. Lin, "Boundary of the numerical range of an operator", Canad. Math. Bulz. 17 (1975), 689-692.

[12] J.G. Stampfli, "Extreme points of the numerical range of a hyponormal operator", Michigan Math. J. 13 (1966), 87-89.

[13] J.G. Stampfli, "The norm of a derivation", Pacific J. Math. 33 (1970), $737-747$.

Federal University of Technology,

P.M.B. 1526,

Owerri,

IMO State,

Nigeria. 